\title{
In vivo immunotoxicity of perfluorooctane sulfonate in BALB/c mice: Identification of T-cell receptor and calcium-mediated signaling pathway disruption through gene expression profiling of the spleen
}

\author{
Qi-Yan Lv, Bin Wan*, Liang-Hong Guo**, Yu Yang, Xiao-Min Ren, Hui Zhang \\ State Key Laboratory of Environmental Chemistry and Ecotoxicology, Research Center for Eco-Environmental Sciences, Chinese Academy of Sciences, Beijing, \\ 10085, China
}

\section{A R T I C L E I N F O}

\section{Article history:}

Received 17 April 2015

Received in revised form

5 June 2015

Accepted 30 July 2015

Available online 20 August 2015

\section{Keywords:}

PFOS

Immunotoxicity

Gene expression profile

Calcium ion influx

T-cell receptor signaling

\begin{abstract}
A B S T R A C T
Perfluorooctane sulfonate (PFOS) is a persistent organic pollutant that is used worldwide and is continuously being detected in biota and the environment, thus presenting potential threats to the ecosystem and human health. Although PFOS is highly immunotoxic, its underlying molecular mechanisms remain largely unknown. The present study examined PFOS-induced immunotoxicity in the mouse spleen and explored its underlying mechanisms by gene expression profiling. Oral exposure of male BALB/c mice for three weeks followed by one-week recovery showed that a $10 \mathrm{mg} / \mathrm{kg} / \mathrm{day}$ PFOS exposure damaged the splenic architecture, inhibited T-cell proliferation in response to mitogen, and increased the percentages of $\mathrm{T}$ helper $\left(\mathrm{CD}^{+}{ }^{+} \mathrm{CD} 4^{+}\right)$and cytotoxic $\mathrm{T}\left(\mathrm{CD}^{+}{ }^{+} \mathrm{CD}^{+}\right)$cells, despite the decrease in the absolute number of these cells. A delayed type of PFOS immunotoxicity was observed, which mainly occurred during the recovery period. Global gene expression profiling of mouse spleens and QRT-PCR analyses suggest that PFOS inhibited the expression of genes involved in cell cycle regulation and NRF2-mediated oxidative stress response, and upregulated those in TCR signaling, calcium signaling, and p38/MAPK signaling pathways. Western blot analysis confirmed that the expressions of CAMK4, THEMIS, and CD3G, which were involved in the upregulated pathways, were induced upon PFOS exposure. Acute PFOS exposure modulated calcium homoeostasis in splenocytes. These results indicate that PFOS exposure can activate TCR signaling and calcium ion influx, which provides a clue for the potential mechanism of PFOS immunotoxicity. The altered signaling pathways by PFOS treatment as revealed in the present study might facilitate in better understanding PFOS immunotoxicity and explain the association between immune disease and PFOS exposure.
\end{abstract}

(C) 2015 Elsevier Ireland Ltd. All rights reserved.

\section{Introduction}

Perfluoroalkyl acids (PFAAs) are a family of synthetic chemicals that have been widely used in industrial and commercial products such as surfactants, lubricants, fabric and paper coatings, firefighting foams, and stain repellants [1]. Among these compounds, perfluorooctane sulfonate (PFOS) is the most studied member due

\footnotetext{
* Corresponding author. State Key Laboratory of Environmental Chemistry and Ecotoxicology, Research Center for Eco-environmental Sciences, Chinese Academy of Sciences, 18 Shuangqing Road, P.O. Box 2871, Beijing, 100085, China.

** Corresponding author. State Key Laboratory of Environmental Chemistry and Ecotoxicology, Research Center for Eco-environmental Sciences, Chinese Academy of Sciences, 18 Shuangqing Road, P.O. Box 2871, Beijing, 100085, China.
}

E-mail addresses: binwan@rcees.ac.cn (B. Wan), LHGuo@rcees.ac.cn (L.-H. Guo). to its extensive distribution [2], long half-life of elimination (about 5.4 years in humans) [3], and bioaccumulation. As a persistent organic pollutant, PFOS has been continually detected in various environmental and biological samples, including humans $[2,4,5]$, even after its voluntary phase-out, probably due to its stability and the biotransformation of its precursor compounds (such as fluorotelomer alcohols) that are still produced in large quantities [6]. Humans are exposed to PFAAs mainly through oral consumption and inhalation [7], which include dietary intake [8], dirty air, and indoor dust ingestion [9], as well as lactational transfer [10]. The serum PFOS concentration of occupational exposure workers ranges from 145 to $3490 \mathrm{ng} / \mathrm{mL}$ [3]; the highest PFOS reported in blood is $12,830 \mathrm{ng} / \mathrm{mL}$ [11] and in the general population, the serum PFOS concentration was found to be within the range of 0.5-92.9 ng/mL [12]. Epidemiological data showed that PFAA 
(including PFOS) exposure was associated with various adverse health effects, including immunodeficiency such as lower antibody responses to childhood immunizations [13] and juvenile asthma [14]. The contamination of PFOS presents potential threats to the ecosystem and human health, thus raising great concerns.

Lines of evidence obtained from animal models strongly suggest that PFOS is immunotoxic [15]. Oral PFOS exposure induces lymphoid organ atrophy, alterations in lymphoid cellular composition, and changes in de novo antibody synthesis, which in turn could lead to a decrease in host resistance to influenza A [16-18]. PFOS exposure has also been shown to decrease the number of Tcell subpopulations and depress the natural killer cell activity of cells in the spleen of adult male C57BL/6 mice [19,20]. PFOS also causes apoptosis in splenocytes and thymocytes [21,22], and interfere with the release of pro-inflammatory cytokines such as TNF- $\alpha$, interleukin (IL)-1 $\beta$, IL-2, IL-4, and IL-6, after short-term or subchronic PFOS exposure [22-24].

The reported side effects of PFOS on the immune system has resulted in a few studies that addressed alterations of signaling transduction in immune cells, although the underlying molecular mechanism of these effects remain unclear. Previous mechanistic studies using non-immune cell models might provide invaluable clues on the mechanisms of PFOS immunotoxicity. For example, the studies based on neuron and liver models indicated that PFOS could disrupt the peroxisome proliferator receptor (PPAR) $\alpha$-mediated pathway, resulting in disruption of lipid metabolism, inflammation, and inhibition of energy homeostasis and cell differentiation [25-27]. Some other studies indicated that PPAR $\alpha$-independent pathways might also be involved in PFOS toxicity because the toxic effects still occurred even in PPAR $\alpha$-null models $[23,28,29]$. Alternatively, these alterations might merely pose an indirect stress effect on immune cells [15].

In the immune system, the spleen plays an important role in initiating immune responses to blood-borne antigens through humoral and cell-mediated pathways [30]. Its dysfunction is usually associated with diminished responsiveness to antigens and increased susceptibility to infection [31]. The bioaccumulation of PFOS in the spleen has been previously reported in rodents [17,32], and several studies have shown that PFOS-induced immunologic outcomes in the spleen involve reduction in weight, structural damages, change in cellular composition, and inflammation [19,33]. To date, information on the underlying mechanisms of these effects is limited. Therefore, in the present study, we hypothesized that PFOS exposure causes damage to the spleen and interferes with multiple pathways that are related to immune cell signaling transduction. Due to the complexity of PFOS toxicity, multiple mechanisms might be involved, but pathways are usually intermingled and crosstalk between each other. The microarray technique has the advantages of taking a snapshot of the expression of thousands of genes in parallel and generating a large amount of data that can potentially provide systems-level information regarding the underlying mechanisms [34]. To test the hypothesis, we orally exposed BALB/c mice to PFOS at levels ranging from 0 to $210 \mathrm{mg} / \mathrm{kg}$ total administered dose (TAD), which was comparable to that utilized by Wang et al. [33] and Zheng et al. [19] (0-280 mg/kg TAD), for a period of three weeks, which was followed by one week of recovery. The one week recovery was set to observe the reversibility, retardance or incubation period for PFOS-induced immunotoxic effects, as PFOS was shown to bio-accumulate/persist in living bodies. The toxicological effects of PFOS on the spleen were first measured, and then the microarray technique was employed for gene expression profiling of the mouse spleen to explore its underlying mechanism.

\section{Materials and methods}

\subsection{Chemicals}

Perfluorooctane sulfonic acid potassium salt (PFOS) (CAS number, 2795-39-3; purity > 98\%) and concanavalin A (Con A) was purchased from Sigma-Aldrich (St. Louis, MO, USA). PFOS was prepared in double-distilled $\mathrm{H}_{2} \mathrm{O}$ with $0.5 \%$ Tween-20 [33]. Con A was dissolved in DMSO.

\subsection{Animals}

Male BALB/c mice (4-5 weeks of age) were purchased from the Department of Laboratory Animal Science, Peking University (Beijing, China) and bred and housed under pathogen-free conditions at the animal care facility. All of the animal experiments conformed to the principles of care and use of laboratory animals and were approved by the Institutional Animal Care and Use Committee of Peking University. The mice were randomly distributed into four groups, with each group consisting of 28 mice. The four groups of mice were orally exposed to $0,2.5,5$, and $10 \mathrm{mg} / \mathrm{kg} /$ day PFOS, respectively, via gastric gavage, and the vehicle control group $(0 \mathrm{mg} / \mathrm{kg} / \mathrm{day})$ was treated with an equal volume of $\mathrm{ddH}_{2} \mathrm{O}$ (with $0.5 \%$ Tween-20). The treatment continued for 3 weeks, followed by a one-week recovery period. The mice in each group were sacrificed by cervical dislocation every 7 days and the spleens were immediately isolated, weighed, and divided into three portions for the following treatments: one portion was fixed in $4 \%$ paraformaldehyde for histological examination (see in Supplementary materials.), the second portion was frozen and stored at $-80^{\circ} \mathrm{C}$ for RNA and protein extraction, and the third was immediately made into a single-cell suspension and red blood cells were lysed for lymphocyte phenotyping and lymphoproliferation analyses. Additionally, a hemocytometer was used to count the number of splenocytes in cell suspension and Trypan blue exclusion was used to assess cell viability, which was always $>90 \%$.

\subsection{Lymphocyte phenotyping}

The T lymphocyte composition of the spleens was analyzed on a BD LSRII flow cytometer (BD Biosciences, San Jose, CA, USA) by using a Mouse T Lymphocyte Subset Antibody Cocktail (BD Pharmingen, San Diego, CA, USA). The single-cell suspension of the splenocytes $\left(1 \times 10^{7}\right.$ nucleated cells/mL PBS) was incubated with antibodies for $30 \mathrm{~min}$ at $4{ }^{\circ} \mathrm{C}$ in the dark. The specific details of this experiment are presented in the Supplementary materials. Isotypic antibody controls were used to set up gates for the T lymphocyte subset $\left(\mathrm{CD}^{+}, \mathrm{CD}^{+} / \mathrm{CD}^{+}\right.$, and $\left.\mathrm{CD}^{+}\right)$phenotyping. The typical gate setting and quadrant analyses are shown in Supplementary Fig. S1. Data were represented as the percentage of the total number of cells. The absolute number of particular cell population was measured and expressed as the ratio of cell number in treatment group after normalization against that in control group.

\subsection{Lymphoproliferation assay}

The single-cell suspension was adjusted to a density of $2 \times 10^{6}$ nucleated cells/mL in RPMI-1640 medium containing 10\% heatdeactivated FBS (cRPMI-1640) and dispensed into a 96-well plate ( $100 \mu \mathrm{L} /$ well) containing triplicate wells of either $5 \mu \mathrm{g} / \mathrm{mL}$ Con A or cRPMI-1640 (as unstimulated wells). The final volume of each well was $200 \mu \mathrm{L}$. The plates were incubated for $72 \mathrm{~h}$ at $37{ }^{\circ} \mathrm{C}$ in a humidified 5\% $\mathrm{CO}_{2}$-air mixture. After incubation, $10 \mu \mathrm{L}$ MTT was 
added into each well, the plate was incubated for $4 \mathrm{~h}$, followed by the addition of $100 \mu \mathrm{L}$ of DMSO to dissolve the formazan crystals. The microplate was then read on a microplate reader (ThermoVarioskan, MA, USA) at a wavelength of $490 \mathrm{~nm}$.

\subsection{Total RNA isolation and microarray analysis}

The spleens of mice in the $10 \mathrm{mg} / \mathrm{kg} / \mathrm{day}$ group at week 3 were used for microarray analysis. Total RNA was extracted using the Trizol reagent (Invitrogen, Carlsbad, CA, USA). The Agilent 2100 Bioanalyzer System (Agilent Technologies, Inc., Waldbronn, Germany) was used to assess the quality and quantity of purified total RNA. Only the samples that passed the RNA quality test were used in the subsequent assays. The GeneChip ${ }^{\circledR}$ Mouse Genome 4302.0 Array (Affymetrix Inc., Santa Clara, CA, USA) was used, and the experiments were performed according to standard procedures. More details can be found in the Supplementary Materials. Three biologic replicates were independently performed for both treatment and control groups (i.e., three sets of microarray data were separately collected from three spleens in each group). Data are deposited at Gene Expression Omnibus (GEO ID: GSE50001).

\subsection{Microarray data analysis and bioinformatics}

Fold change in gene expression was determined from the nonlog-transformed signal data after correction and normalization by using Ingenuity ${ }^{\circledR}$ iReport ${ }^{\mathrm{TM}}$. Gene expression fold change $>1.5$ or $<-1.5$ and $p<0.05$ was set and considered significantly different from that observed in the control. Simultaneously, pathway analysis was performed, and the details can be found at the company website [http://www.ingenuity.com/products/ireport (ID: 2794)]. We also used the NCBI Database for Annotation, Visualization and Integrated Discovery (DAVID 6.7, http://david.abcc.ncifcrf.gov) for gene ontology analysis. A modified Fisher's exact test [35] was used to measure gene set enrichment. $P$-values $<0.05$ and an enrichment factor $>2$ were considered significant.

\subsection{Quantitative real-time $P C R(q R T-P C R)$ analysis}

qRT-PCR was perform by using a RevertAid First Strand cDNA Synthesis Kit (Fermentas China Co. Ltd, Shenzhen, China) and a
GoTaq ${ }^{\circledR}$ qPCR Master Mix (Promega, Madison, WI, USA) in a Mx3005P real-time QPCR system (Stratagene, La Jolla, CA, USA), following the manufacturer's protocols. The primers we used are listed in Table 1. Data quality control and analyses were performed according to the method described by Pfaffl and coworkers [36], and detailed information can be found in the Supplementary Material. Examination of the expression level of the housekeeping gene, glyceraldehyde-3-phosphate dehydrogenase (GAPDH), in the microarray data showed no significant change between the control and treatment samples. Therefore, GAPDH was used as endogenous control.

\subsection{Western blot analysis}

The spleens were lysed with RIPA lysis buffer, and samples were separated by SDS-PAGE with $10 \%$ (15\% for CD3G protein) separation gel for protein detection. The samples were transferred onto PVDF membrane $(0.45 \mu \mathrm{m})$, and the membranes were then blocked with $5 \%$ non-fat milk and washed in TBST (containing 0.1\% Tween-20). The membranes were incubated with primary antibodies $(1: 1000$ dilution) at $4{ }^{\circ} \mathrm{C}$ overnight, and after TBST washing, the membranes were incubated with the corresponding HRP-conjugated secondary antibody (1:5000 dilutions) for $1 \mathrm{~h}$ at room temperature. The membrane was further developed with the ECL Plus Western blotting detection system (Millipore Corporation, Billerica, MA, USA). The relative abundance of each protein was measured by using the Gel-Pro Analyzer software (Version 4.0, Media Cybernetics, Inc., Bethesda, MD, USA). $\beta$-actin was used as internal control.

\subsection{Measurement of intracellular free $\mathrm{Ca}^{2+}$ concentration $\left(\left[\mathrm{Ca}^{2+}\right]_{i}\right)$}

The single-cell suspension of splenocytes was grown on 96well plates at a cell density of $2 \times 10^{6}$ nucleated cells $/ \mathrm{mL}$ for overnight $(16 \mathrm{~h})$ and then loaded with Fura-2/AM $(5 \mu \mathrm{M})$ for $45 \mathrm{~min}$ in cRPMI-1640 at $37^{\circ} \mathrm{C}$ prior to PFOS exposure. The increase in intracellular $\left[\mathrm{Ca}^{2+}\right]_{i}$ was expressed as the fluorescence intensity ratio measured at $340 \mathrm{~nm}$ and $380 \mathrm{~nm}$ excitation wavelengths (F340/F380), as it is proportional to calcium ion concentrations. F340/F380 ratiometric measurement of emission at $510 \mathrm{~nm}$ was obtained after $5 \mathrm{~h}$ and $24 \mathrm{~h}$ PFOS exposure. The

Table 1

Comparison of the gene expression ratios $(\mathrm{T} / \mathrm{C})$ as determined by microarray and quantitative RT-PCR analyses. Results were generated from triplicates $(\mathrm{n}=3$ ).

\begin{tabular}{|c|c|c|c|c|}
\hline Gene symbol & GenBank accession no. & RT-PCR ${ }^{\mathrm{a}}$ & Array $^{\mathrm{b}}$ & Sequence of primers used in RT-PCR \\
\hline CACNA1A & NM_007578.3 & 1.54 & 1.7 & $\begin{array}{l}\text { F: } 5^{\prime} \text {-tcctgtgctcgtttctgatg-3' } \\
\text { R: } 5^{\prime} \text {-acacgcacgtattcatccaa-3' }\end{array}$ \\
\hline CAMK 4 & NM_009793.3 & 1.9 & 1.7 & $\begin{array}{l}\text { F: 5'-agcacattcaaaccaccaca-3' } \\
\text { R: } 5^{\prime} \text {-ggactcagagatccgtctgc-3' }\end{array}$ \\
\hline$C D 3 G$ & NM_009850.2 & 1.71 & 1.5 & $\begin{array}{l}\text { F: } 5^{\prime} \text {-atatctcattgcgggacagg- } 3^{\prime} \\
\text { R: } 5^{\prime} \text {-ccctgagtcctgctgagttc- } 3^{\prime}\end{array}$ \\
\hline Themis & NM_178666.5 & 1.13 & 1.6 & $\begin{array}{l}\text { F: 5'-caccgaagacacaacaatgg-3' } \\
\text { R: } 5^{\prime} \text {-acagcattcattgccaaaca-3' }\end{array}$ \\
\hline МАРК12 & NM_013871.3 & 4.11 & 1.6 & $\begin{array}{l}\text { F: } 5^{\prime} \text {-gagacttgaagcctggcaac- } 3^{\prime} \\
\text { R: } 5^{\prime} \text {-ccgggttaccacatatcctg-3' }\end{array}$ \\
\hline МАРЗК5 & NM_008580.4 & 1.3 & 1.5 & $\begin{array}{l}\text { F: } 5^{\prime} \text {-aagaggcttgctggcataaa-3' } \\
\text { R: } 5^{\prime} \text {-tgcctgtggctctcctagtt- } 3^{\prime}\end{array}$ \\
\hline E2F1 & NM_009692.3 & 0.15 & 0.5 & $\begin{array}{l}\text { F: 5'-agcctagggattcagggtgt-3' } \\
\text { R: } 5^{\prime} \text {-cagagggtatggatcgtgct-3' }\end{array}$ \\
\hline$C D C 25 A$ & NM_007658 & 0.75 & 0.67 & $\begin{array}{l}\text { F: 5'-gggaagcatcaggatttgaa-3' } \\
\text { R: } 5^{\prime} \text {-cacccttgatgtgacctcct- } 3^{\prime}\end{array}$ \\
\hline CDK1 & NM_007659.3 & 0.14 & 0.37 & $\begin{array}{l}\text { F: } 5^{\prime} \text {-agctctgggcactcctaaca- } 3^{\prime} \\
\text { R: } 5^{\prime} \text {-ccattttgccagagattcgt- } 3^{\prime}\end{array}$ \\
\hline CDKN1A & NM_007669 & 0.8 & 0.65 & $\begin{array}{l}\text { F: } 5^{\prime} \text {-cacagctcagtggactggaa-3' } \\
\text { R: } 5^{\prime} \text {-accctagaccacaatgcag-3' }\end{array}$ \\
\hline
\end{tabular}

a Gene expression ratios determined by quantitative RT-PCR.

b Gene expression ratios as determined by microarray analyses. F: Forward primer; R: Reverse primer. 
experiment was conducted in the dark.

\subsection{Statistical analyses}

Data were expressed as the mean $\pm \mathrm{SD}$, and the student's $t$-test was used to compare differences between treatment and control groups, with the significance level set at $p<0.05$.

\section{Results}

\subsection{General toxicity and lymphoproliferation assay}

During the exposure week (week 1-3), mice in all groups gained weight steadily over time and no significant difference in body weight was observed among treatment groups and control group (Fig. 1A). But in the recovery period (week 4), the body weight in $10 \mathrm{mg} / \mathrm{kg} /$ day group was lower than that of control. Spleen index also decreased in a dose-dependent manner during the recovery period (Fig. 1B). Histopathological examination showed that PFOS exposure resulted in alterations in spleen architecture (Fig. S2).

In general, Con A-induced T-cell proliferation was not affected during week $1-3$, except for the first week in the $10-\mathrm{mg} / \mathrm{kg} /$ day group, which caused significant $(p<0.05)$ inhibition of T-cell proliferation. Apparent dose-dependent inhibition of T-cell proliferation was observed during the recovery period (week 4), with the ratio decreasing from 1 to 0.85 and 0.84 in $5 \mathrm{mg} / \mathrm{kg} /$ day and $10 \mathrm{mg} /$ $\mathrm{kg} /$ day groups $(p<0.05)$, respectively (Fig. $1 C)$.

\subsection{Lymphocyte phenotyping}

At weeks 2 and 3, the percentage of $\mathrm{CD}^{+}$cells increased from $33.8 \%$ (control, week 2 ) to $48.7 \%$ and $40.7 \%$ (control, week 3 ) to $50.17 \%$ in the $5 \mathrm{mg} / \mathrm{kg} /$ day groups, respectively (Fig. 1D). After recovery, the abundance of $\mathrm{CD}^{+}$cells significantly increased in the $10 \mathrm{mg} / \mathrm{kg}$ /day group, with the percentage increasing from $39.87 \%$ to $52.8 \%$. At week 3 , the abundance of $\mathrm{CD}^{+} \mathrm{CD}^{+}$cells increased in a dose-dependent manner, with the percentage increasing from $8.9 \%$ (control) to $10.8 \%$ and $11.7 \%$, in the $5 \mathrm{mg} / \mathrm{kg} /$ day and $10 \mathrm{mg} / \mathrm{kg} /$ day groups, respectively (Fig. 1E). A similar trend was observed in the $\mathrm{CD}^{+}{ }^{+} \mathrm{CD} 4^{+}$cells, with the percentage increasing from $18.4 \%$ (control) to $23.7 \%, 25.7 \%$, respectively (Fig. $1 \mathrm{~F}$ ). Although the percentage increased, the absolute number of $\mathrm{T}$ cell subpopulations decreased in the $5 \mathrm{mg} / \mathrm{kg} / \mathrm{day}$ and $10 \mathrm{mg} / \mathrm{kg} /$ day groups in a dose-dependent manner during the recovery period (Fig. 1)G, H, and I.
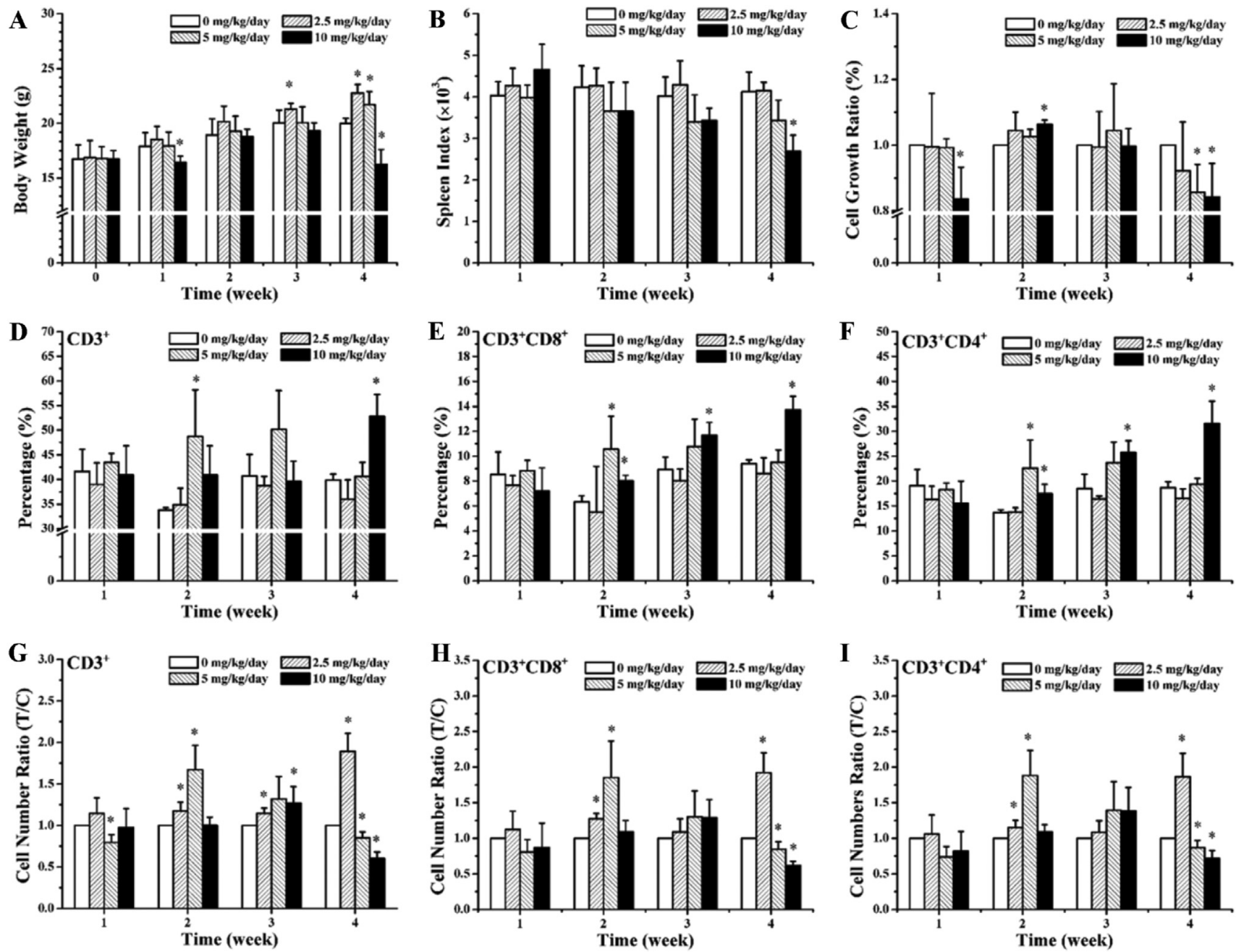

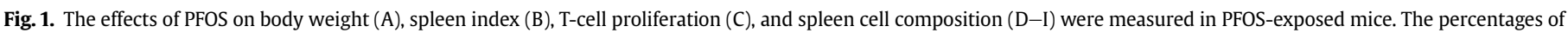

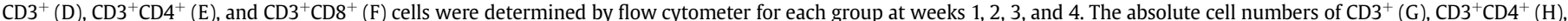

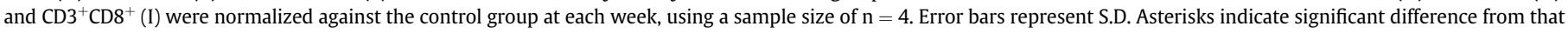
observed in the control, at a significance level of ${ }^{*} p<0.05$. 


\subsection{Gene expression profile and bioinformatics analyses}

To further explore the underlying mechanisms of the PFOS sideeffects in the spleen, gene expression profiling was conducted using microarray analyses. Based on the criteria of significance, changes in the expression of 1327 genes (4\% of all analyzed genes) in the 10$\mathrm{mg} / \mathrm{kg} /$ day group were observed. Among these, 239 (0.8\%) were upregulated, and 1088 (3.2\%) were downregulated. Using the DAVID online tool, we further examined the functional distribution and category enrichment of the 1327 differentially expressed genes (DEGs) based on gene ontology terms. Of the gene list, 924 (69.6\%) genes were mapped to the biological processes of the DAVID knowledge base. Sixteen biological processes were enriched (Fig. 2A), with the majority related to cell cycle/cell cycle control (15.7\%, 145/924), DNA metabolism (7.5\%), mitosis (5.8\%), and DNA replication (4.3\%), with respect to the whole mouse genome reference dataset. The enrichment analysis showed that in addition to DNA metabolism (enrichment factor, 3.7), replication (5.6), recombination (4.9), and chromosome segregation (3.9), a set of processes including porphyrin metabolism (6.8), MHCII-mediated immunity (3.7), hematopoiesis (3.2) and lipid and fatty acid transport (2.1) were also enriched, suggesting that these cellular processes were affected by PFOS. GO analysis showed that 15 molecular functions were significantly enriched, including alipoprotein (enrichment factor, 7.9), replication origin-binding protein (10.6), DNA-directed DNA polymerase (6.6), DNA helicase (4.4), and kinase activator (2.6) (Fig. 2B).

We then used Ingenuity tools to perform pathway analysis of the DEGs to gather all the information on the molecular mechanism of PFOS-induced immunotoxicity. A total of 501 genes were mapped in the Ingenuity knowledge base, and based on criteria ( $p<0.05$ and $>5$ genes in the pathways), 66 pathways were significantly enriched in the treatment group. The full list can be found in Supplementary Table S1. A few pathways were enriched because the same DEGs populated multiple pathways.

Examination of changes in gene expression patterns of these pathways showed that cell cycle-related pathways (including Cell Cycle Control of Chromosomal Replication, Role of CHK Proteins in Cell Cycle Checkpoint Control, Cell Cycle G2M DNA Damage Checkpoint Regulation, Cyclins and Cell Cycle Regulation, and Cell Cycle G1S Checkpoint Regulation) were most significantly downregulated by PFOS treatment (Fig. $3 \mathrm{~A}$ ). The pathways related to Tcell receptor (TCR) and signaling (primary immunodeficiency signaling, iCOS-iCOSL signaling in T helper cells, calcium-induced T lymphocyte apoptosis, and OX40 signaling pathway) were significantly upregulated, along with the downregulation of the antiproliferative role of TOB in the T cell signaling pathway (Fig. 3B). The p38 MAPK signaling pathway represented a more complicated pattern of change in expression, with MAPK12 and MAP3K5 upregulated, whereas MAP2k3 was downregulated (Fig. 3C). In addition, gene (such as GSTM3 and MGST3) expression in NRF2mediated oxidative stress response decreased (Fig. 3D). The
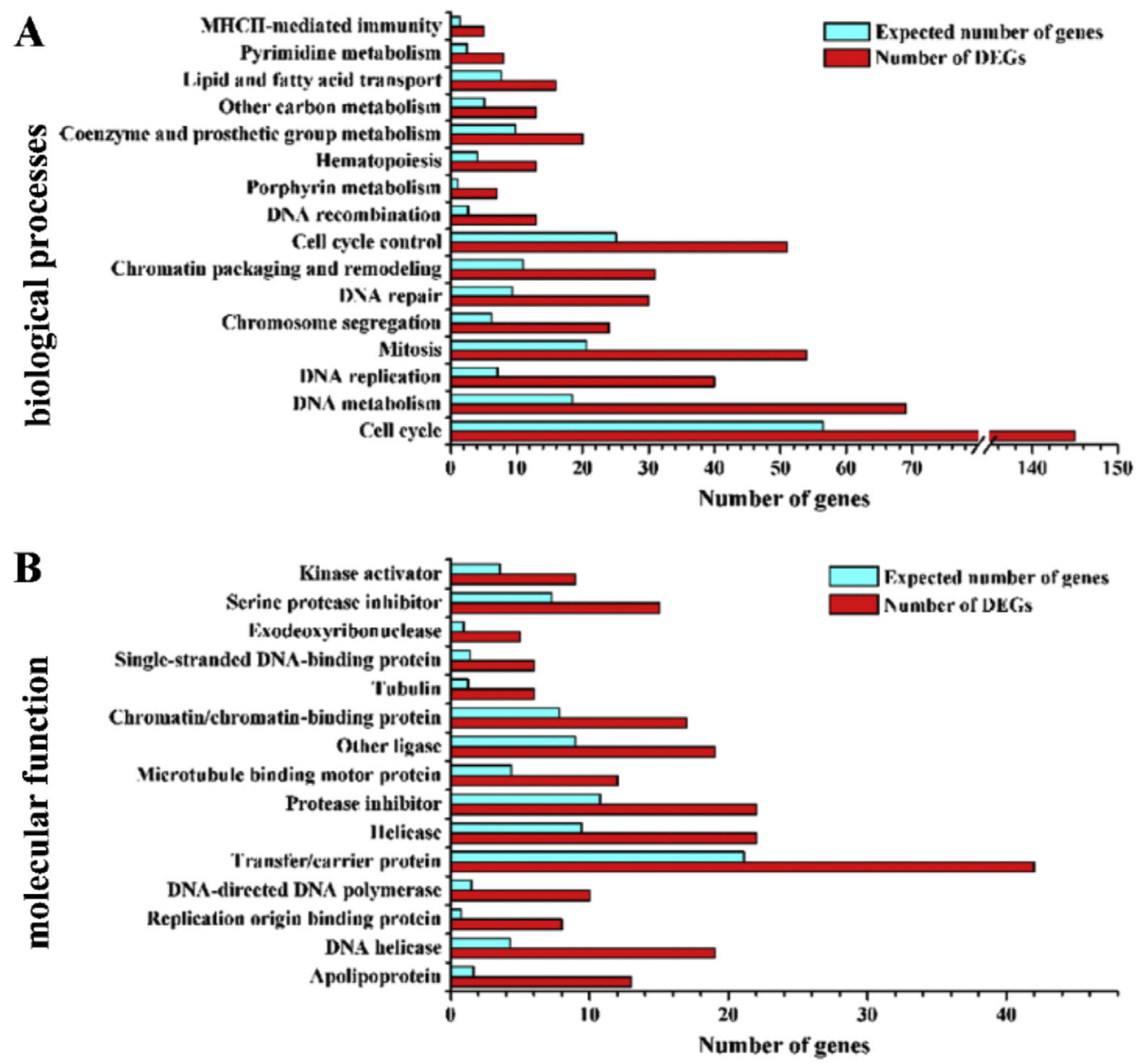

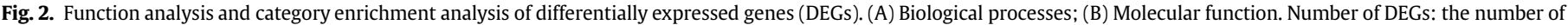

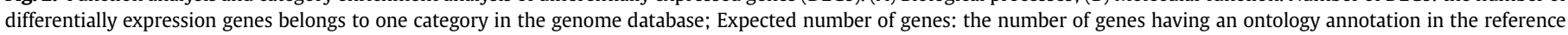
database. 


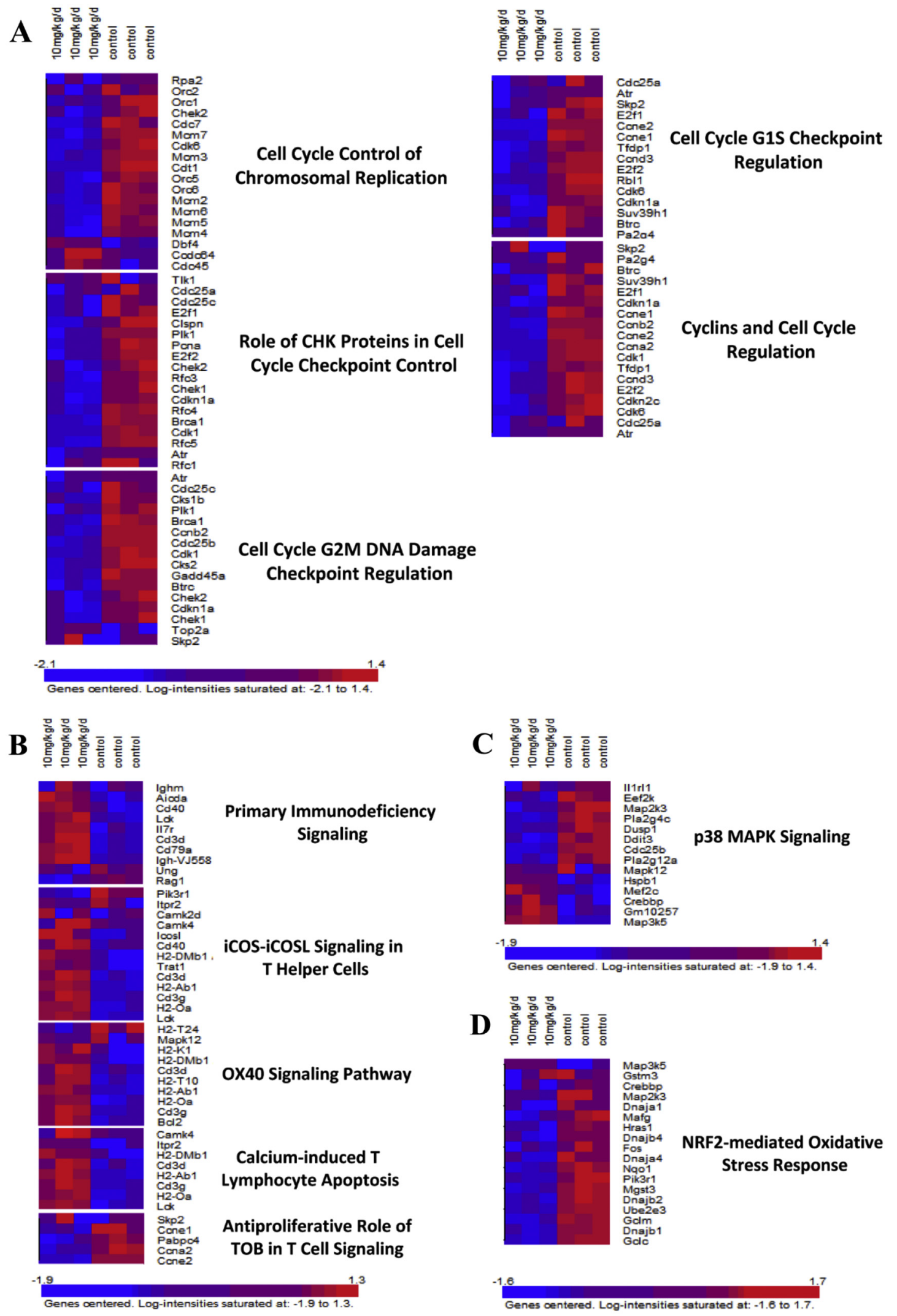

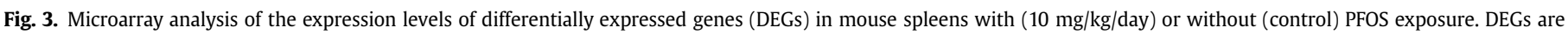
involved in (A) cell cycle control related pathways, (B) T-cell receptor (TCR) and signaling, (C) p38 MAPK signaling, and (D) NRF2-mediated oxidative stress response. 
corresponding information on these pathways is presented in Table S2. These results showed that PFOS had an important effect on these pathways. Among them, the ones related to T-cell development and signaling are of particular biological significance in terms of the immunotoxicity of PFOS because $\mathrm{T}$ cells play an important role in cell-mediated immunity.

To further confirm the results of microarray analyses, we selected a few key genes involved in these pathways and used QRTPCR to quantify changes in levels of expression. These genes were determined to be related to: TCR signaling (CD3G and Themis), calcium ion regulation and signaling, including CAMK4 and CAC$N A 1 A$, MAPK signaling (MAPK12 and MAP3K5), and cell cycle regulation (E2F1, CDC25A, CDK1, and CDKN1A). A similar trend in was observed between QRT-PCR and the microarray data (Table 1).

\subsection{Western blot assay and intracellular free $\mathrm{Ca}^{2+}$ concentration}

It is well known that $\mathrm{Ca}^{2+}$ plays an important role in the proliferation of immune cells in response to foreign antigens [37]. Therefore, interference of $\mathrm{Ca}^{2+}$ might be involved in the immunotoxicity of PFOS. Based on above results, we measured the protein expression levels of Themis, CD3G, and CAMK4, due to the fact that they are closely related to TCR signaling and calcium signaling. In general, the change in the expression of these proteins was more prominent in week 4 than in week 3, and was dose-dependent. For example, the expression level of Themis increased by 1.2- and 1.5fold at weeks 3 and 4 in the $10 \mathrm{mg} / \mathrm{kg} /$ day group, respectively. CAMK4 was significantly $(p<0.05)$ upregulated at week 4 in the 5 (2.4-fold) and 10 (2.5-fold) $\mathrm{mg} / \mathrm{kg} /$ day groups. Similarly, the level of CD3G expression changed by 0.9 - and 1.9 -fold in the 5 and $10 \mathrm{mg} /$ $\mathrm{kg}$ /day groups, respectively, at week 3 . A more prominent dose-response was observed at week 4, with the expression levels increasing by 1.5 - and 2.5-fold, respectively (Fig. 4).

In addition, based on microarray analysis, which shows that
PFOS could modulate $\mathrm{Ca}^{2+}$-induced $\mathrm{T}$ cell apoptosis pathway, we further measured the $\left[\mathrm{Ca}^{2+}\right]_{i}$ of splenocytes after acute PFOS exposure to illustrate the effects of PFOS on calcium homoeostasis. A series of acute PFOS exposure at concentrations $>25 \mu \mathrm{M}$ (approximately $12.5 \mu \mathrm{g} / \mathrm{mL}$, the highest occupational plasma level) was used to simulate a low concentration long-term effect. Cell viability was not affected by these treatments for as long as $24 \mathrm{~h}$ (Fig. S3). As shown in Fig. 5, Con A (positive control) induced an increase in $\left[\mathrm{Ca}^{2+}\right]_{\mathrm{i}}$ by 1.45 -fold. After $5 \mathrm{~h}$ of PFOS exposure, $\left[\mathrm{Ca}^{2+}\right]_{\mathrm{i}}$ increased in a concentration-dependent manner, with the F340/ F380 ratio increasing from 1.19 (control) to $1.23,1.32$, and 1.32 in 100,200 , and $500 \mu \mathrm{M}$ treatment groups, respectively (Fig. 5A). A similar trend was observed after $24 \mathrm{~h}$ exposure, with higher ratios (Fig. 5B).

\section{Discussion}

The PFOS concentration in the general population and in nature was at the ng/mL level. Recently, an investigation on dust around fluorochemical plant revealed extremely high PFOS concentrations, ranging from 2.68 to $18,486 \mathrm{ng} / \mathrm{g}$ (or $18.486 \mathrm{mg} / \mathrm{kg}$ ) [38], indicating that the occupational situation apparently caused much higher exposure levels of PFOS to workers, the resulting serum concentration could reach as high as $>10 \mu \mathrm{g} / \mathrm{mL}$ level [3,11]. A study employing 5-40 mg/kg/day exposure treatment showed that PFOS could induce splenic atrophy and lymphocyte disorders [19]. Studies using a similar range of exposure dosage (5-20 mg/kg/day) for 7-14 days or a 10-day treatment with dietary doses (1\%$0.001 \%$, PFOS $\mathrm{w} / \mathrm{w}$ ) revealed the destructive effect of PFOS on splenic structure, changes in inflammatory response, and cytokine production $[20,28,33]$. Therefore, we used up to $2.5-10 \mathrm{mg} / \mathrm{kg} / \mathrm{day}$ as the treatment to simulate the high occupational exposure scenario. Several previous studies based on similar exposure framework revealed that exposure of male C57BL/6 mouse to 50,125, 140 ,
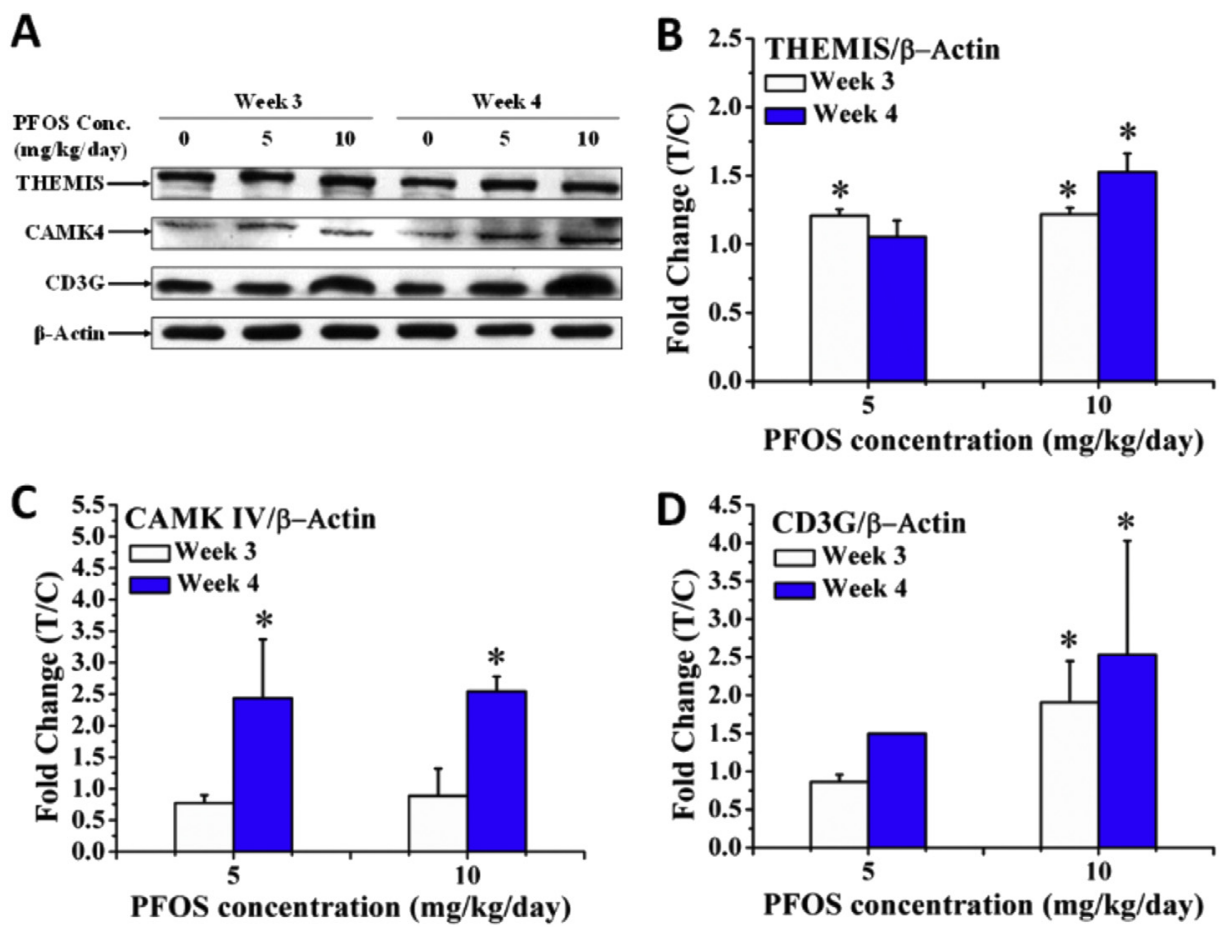

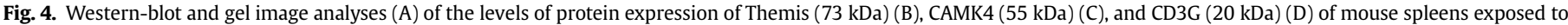

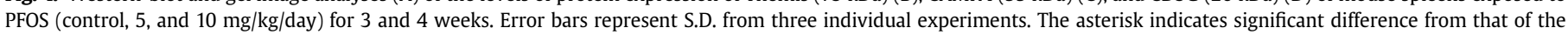
control at a significance level of $* p<0.05$. 

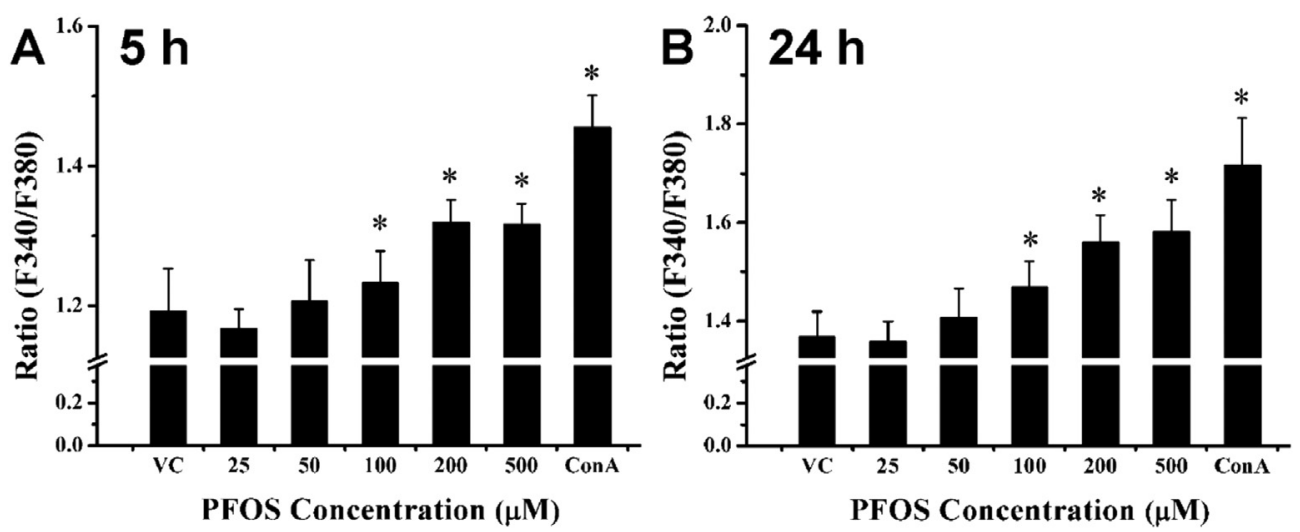

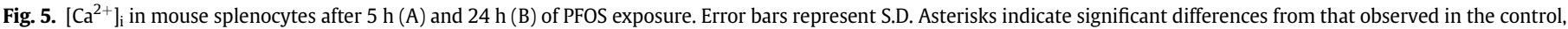
at a significance level of ${ }^{*} p<0.05$.

200 and $280 \mathrm{mg} / \mathrm{kg}$ TAD resulted in serum PFOS concentrations of 51.7-59.7 mg/L [21,24], 120.7 mg/L [16], $250 \mathrm{mg} / \mathrm{L}$ [20], $340 \mathrm{mg} / \mathrm{L}$ [39], and $338 \mathrm{mg} / \mathrm{L}$ [19], respectively. The TADs used in our experiment were 52.5 (lowest) and $210 \mathrm{mg} / \mathrm{kg}$ (highest) and therefore the lowest serum PFOS concentration would be around 50-60 mg/ $\mathrm{L}$ and the highest concentration would be around $340 \mathrm{mg} / \mathrm{L}$ at the end of exposure, respectively. In the note of different mouse strains (BALB/c in this study vs. C57BL/6), the measurement should be included in our future works and more studies are needed to analyze the effects of long-term exposure to lower dosages of PFOS on immune system. In the present study, when mice were exposed to comparable dosages of PFOS (2.5-10 mg/kg/day), the results showed that although PFOS caused atrophy of the spleen and a decrease in the absolute number of T-cell subpopulations, the percentages of these cells $\left(\mathrm{CD}^{+}{ }^{+} \mathrm{CD} 4^{+}\right.$and $\left.\mathrm{CD}^{+}{ }^{+} \mathrm{CD}^{+}\right)$increased. The result is consistent with the findings of a few studies that described interference of perfluorinated chemicals in $\mathrm{T}$ lymphocyte subsets by decreasing the absolute number of these cells in adult male mice $[16,40]$. In addition, inhibition of the T-cell response to a mitogen was suggestive of the anergy of this cell population after PFOS exposure.

During PFOS exposure (weeks 1-3), majority of the toxic effects on spleens were not significant until the recovery week (week 4), indicating that the spleens underwent a series of molecular events during the exposure, which led to a delayed type of toxicity to PFOS. Our research group is particularly interested in the alterations occurring right before the cessation of PFOS exposure. Therefore, we determined the global gene expression profiles of spleens in highest treatment group ( $10 \mathrm{mg} / \mathrm{kg} /$ day) at the time point of week 3. Comparison of the expression patterns of the control and treatment groups showed that a variety of cell cycle-related pathways, including cell cycle control of chromosomal replication, G1/S checkpoint regulation, G2/M DNA damage checkpoint regulation, CHK proteins in cell cycle checkpoint control, and cyclins and cell cycle regulation were inhibited (Fig. 3A). Similar results were previously reported in PFOS-exposed bottlenose dolphin skin cells and splenocytes, thus indicating regulation of cell cycle progression and cell proliferation using stage-specific checkpoints [21,41]. The main mechanism of the cell cycle checkpoints involves the regulation of the cyclin-dependent kinase (CDK) activities by CDC25A [42]. CDKs are protein kinases that modulate the activity of its substrates, which include the transcription factors E2F (E2F1) and its regulator $\mathrm{Rb}$, thereby controlling cell cycle progression. The decrease in CDK1 activity results in a delay in cell cycle progression in human $\mathrm{T}$ lymphocytes, which in turn leads to the impairment of the proliferative response of $\mathrm{T}$ lymphocytes to mitogenic stimulation [43].
Therefore, the downregulation of $C D C 25 A, C D K 1$, and E2F1 by PFOS exposure could inhibit cell cycle progression, resulting in the decrease in the rate of $\mathrm{T}$ lymphocyte proliferation upon Con A stimulation, as shown in Fig. 1C. The massive deregulation of cell cycle checkpoint pathways could also contribute to the decrease in the number of T cells in the spleen (Fig. 1)H and I.

More importantly, several pathways directly related to the generation of immune cell responses were identified to be modulated by PFOS, including primary immunodeficiency signaling, iCOS-iCOSL signaling in helper T cells, anti-proliferative role of TOB in T cell signaling, OX40 signaling pathway, and calcium-induced T lymphocyte apoptosis. These pathways converge at the MHC-IIand TCR-mediated immunity and calcium signaling cascade as a group of genes that involve calcium regulation (such as CAMK4 and CAMK2d), and the MHC-II complex (i.e., H2-T24, H2-K1, H2-DMB1, $H 2-T 10, H 2-A b 1$, and $H 2-O A$ ) and TCR (i.e., CD3G, CD3d, and $L C K$ ) were present in multiple pathways and were significantly upregulated at the transcriptional or protein levels (Figs. 3B and 4). Calcium ions are involved in various important biological processes, and calcium-mediated signaling pathways play crucial roles in a wide range of cellular events, including cell proliferation and differentiation, apoptosis, and autophagy [44]. The effects of PFOS on calcium channel downstream signaling have been reported in the nervous system, revealing a molecular mechanism of its neurotoxicity [44]. In the present study, calcium signaling in lymphocytes was determined to be modulated by PFOS, and an increase in intracellular $\mathrm{Ca}^{2+}$ concentration was detected after exposure to this particular reagent (Fig. 5). Prolonged and relatively higher $\mathrm{Ca}^{2+}$ concentrations could activate a sequence of downstream signaling pathways and disrupt the interactions between T-cells and antigenpresenting cells in inducing alterations in cytoskeletal organization and motility [45], developmental arrest, unresponsiveness, and apoptosis of $\mathrm{T}$ lymphocytes [46,47]. Therefore, the calcium signaling pathway and disturbances in free intracellular $\mathrm{Ca}^{2+}$ concentrations might be involved in the mechanism of PFOS immunotoxicity.

Themis is expressed in spleen, regulates calcium influx in cells, and activate TCR signaling during T-cell maturation. Changes in the expression of Themis occur during developmental immaturity [48]. Therefore, the PFOS-induced upregulation of Themis suggests its disruptive effects on the maturation of T-cells, which eventually leads to T-cell anergy. Similarly, CD3G, along with LCK, can form a complex with TCRs. Defects in these genes are associated with T cell immunodeficiency and dysfunction of mature T-cells [49,50]. The order and ratio of $\mathrm{CD} 3$ peptides (i.e., gamma, epsilon, and delta) in association with TCRs are tightly regulated during the maturation of 
T cells, and its disruption can lead to biased T cell proliferation [51]. CAMK4 operates in the calcium-triggered CaMKK-CaMK4 signaling cascade and regulates the activity of transcription activators, by which CAMK4 links T-cell antigen receptor (TCR) signaling to the production of IL-2, IFN-gamma, and IL-4 [52-54], which in turn determines the nature of an immune response. Recently, PFOS was shown to suppress IL-2 production in both human T cell lines and primary T-cells [55], indicating that the disruption of TCR signaling by PFOS exposure might cause the arrest of T-cell proliferation and altered immune responses. Interestingly, Negoro and coworkers [56] showed that the incidence of asthma was caused by an increase in $\left[\mathrm{Ca}^{2+}\right]_{i}$ of regulatory T-cells following TCR activation, with partial loss of regulatory functions, indicating that PFOS-induced calcium influx and TCR activation might be responsible for juvenile asthma, as reported by Dong et al. [14].

In addition, CAMK4 can activate MAPK14/p38 and stimulate transcription of downstream genes [53,57]. MAPK pathways play a crucial role in both adaptive and innate immunities, regulating immune responses [58,59]. Interestingly, our microarray data showed that the p38/MAPK pathway was modulated by PFOS, with the upregulation of stress-activated MAP kinase genes (such as MAPK12 and MAP3K5 in Table 1 and Fig. 3B). It has been reported that PFOS-activated p38/MAPK pathways could induce apoptosis of cerebellar granule cells and zebrafish embryo cells [60,61]. Therefore, the activation of MAPK pathway by PFOS may result in immune cell apoptosis and thus atrophy of the spleen. Alternatively, the pathways can also be activated by oxidative stress [62]. It has been illustrated that PFOS could overwhelm the homeostasis of anti-oxidative systems in orally PFOS-exposed adult male C57BL/6 mice, resulting in ROS overproduction and decreased glutathioneS-transferase (GST), as well as splenocyte apoptosis [22]. The results of our microarray analyses showed that the NRF2-mediated oxidative stress response pathway was inhibited by PFOS exposure, along with the downregulation of GSTM3 and microsomal GST (MGST3), as shown in Fig. 3D, suggesting that the role of oxidative stress in induction of MAPK pathways might not be excluded.

Future studies should investigate the gene expression profiles of the thymus because $T$ cells originate from the bone marrow and mature in the thymus, which are then released to the peripheral tissues. In addition, the thymus has been shown to accumulate PFOS at a similar magnitude as that observed in the mouse spleen [17]. In addition, the effects of PFOS on the thymus have been previously reported [16]. The results of such work could provide more clues on the potential toxic mechanisms of PFOS. Furthermore, the percentage of $\mathrm{T}$ cells generally increased, although the absolute number of T cells decreased in a dose-dependent manner, indicating a severe decrease in other cell populations in the spleen. Therefore, future work should focus on other immune cells using various dosage and time points.

\section{Conclusions}

In summary, the ubiquitous PFOS contamination poses a potential threat to the environment and human health, and its immunotoxicity raises concern particularly when its molecular mechanisms have not been fully established. The present study examined the toxic effects and the underlying mechanisms of PFOS in the BALB/c mouse spleen. The results showed that PFOS induced spleen atrophy, as well as changes in spleen architecture and composition of T-cell subpopulations. T-cell proliferation in response to Con A stimulation was inhibited by PFOS exposure. Most of these effects were not prominent during the exposure period of PFOS until after a week recovery. Microarray analyses showed that during the PFOS exposure, a variety of cell cycle regulation pathways were inhibited, along with the activation of pathways related to T-cell signaling and development and calcium ion influx. The altered signaling pathways by PFOS treatment as revealed by the present study might facilitate in better understanding PFOS immunotoxicity and shed light on the toxic mechanisms and pathogenesis of PFOS exposure.

\section{Conflict of interest statement}

The authors report no conflicts of interest. The authors alone are responsible for the content and writing of the paper.

\section{Acknowledgments}

The National Basic Research Program of China (No. 2011CB936001), the National Natural Science Foundation of China (21277158, 21477146, 21207146, 21377142, 21407168, 21321004), and Chinese Academy of Sciences (XDB14040100, YSW2013A01) supported this research study.

\section{Appendix A. Supplementary data}

Supplementary data related to this article can be found at http:// dx.doi.org/10.1016/j.cbi.2015.07.015.

\section{Transparency document}

Transparency document related to this article can be found online at http://dx.doi.org/10.1016/j.cbi.2015.07.015.

\section{References}

[1] J.P. Giesy, K. Kannan, Global distribution of perfluorooctane sulfonate in wildlife, Environ. Sci. Technol. 35 (7) (2001) 1339-1342.

[2] J.E. Naile, J.S. Khim, S. Hong, J. Park, B.O. Kwon, J.S. Ryu, J.H. Hwang, P.D. Jones, J.P. Giesy, Distributions and bioconcentration characteristics of perfluorinated compounds in environmental samples collected from the west coast of Korea Chemosphere 90 (2) (2013) 387-394.

[3] G.W. Olsen, J.M. Burris, D.J. Ehresman, J.W. Froehlich, A.M. Seacat J.L. Butenhoff, L.R. Zobel, Half-life of serum elimination of perfluorooctanesulfonate, perfluorohexanesulfonate, and perfluorooctanoate in retired fluorochemical production workers, Environ. Health Perspect. 115 (9) (2007) 1298-1305.

[4] V.L. Jaspers, D. Herzke, I. Eulaers, B.W. Gillespie, M. Eens, Perfluoroalkyl substances in soft tissues and tail feathers of Belgian barn owls (Tyto alba) using statistical methods for left-censored data to handle non-detects, Environ. Int 52 (0) (2013) 9-16.

[5] Y.G. Zhao, C.K. Wong, M.H. Wong, Environmental contamination, human exposure and body loadings of perfluorooctane sulfonate (PFOS), focusing on Asian countries, Chemosphere 89 (4) (2012) 355-368.

[6] C.M. Butt, D.C. Muir, S.A. Mabury, Biotransformation pathways of fluorotelomer-based polyfluoroalkyl substances: a review, Environ. Toxicol. Chem. 33 (2) (2014) 243-267.

[7] O. Weiss, G.A. Wiesmuller, A. Bunte, T. Goen, C.K. Schmidt, M. Wilhelm, J. Holzer, Perfluorinated compounds in the vicinity of a fire training area human biomonitoring among 10 persons drinking water from contaminated private wells in Cologne, Germany, Int. J. Hyg. Environ. Health 215 (2) (2012) 212-215.

[8] J.L. Domingo, Health risks of dietary exposure to perfluorinated compounds, Environ. Int. 40 (2012) 187-195.

[9] L. Knobeloch, P. Imm, H. Anderson, Perfluoroalkyl chemicals in vacuum cleaner dust from 39 Wisconsin homes, Chemosphere 88 (7) (2012) 779-783.

[10] A.E. Loccisano, J.L. Campbell Jr., J.L. Butenhoff, M.E. Andersen, H.J. Clewell 3rd, Evaluation of placental and lactational pharmacokinetics of PFOA and PFOS in the pregnant, lactating, fetal and neonatal rat using a physiologically based pharmacokinetic model, Adv. Exp. Med. Biol. 33 (4) (2012) 468-490.

[11] H. Fromme, S.A. Tittlemier, W. Volkel, M. Wilhelm, D. Twardella, Perfluorinated compounds-exposure assessment for the general population in Western countries, Int. J. Hyg. Environ. Health 212 (3) (2009) 239-270.

[12] S. Saikat, I. Kreis, B. Davies, S. Bridgman, R. Kamanyire, The impact of PFOS on health in the general population: a review, Environ. Sci. Processes Impacts 15 (2) (2013) 329-335.

[13] P. Grandjean, E.W. Andersen, E. Budtz-Jørgensen, F. Nielsen, K. Mølbak, P. Weihe, C. Heilmann, Serum vaccine antibody concentrations in children exposed to perfluorinated compounds, JAMA J. Am. Med. Assoc. 307 (4) (2012) 391-397. 
[14] G.H. Dong, K.Y. Tung, C.H. Tsai, M.M. Liu, D. Wang, W. Liu, Y.H. Jin, W.S. Hsieh, Y.L. Lee, P.C. Chen, Serum polyfluoroalkyl concentrations, asthma outcomes, and immunological markers in a case-control study of taiwanese children, Environ. Health Perspect. 121 (4) (2013) 507-513.

[15] J.C. Dewitt, M.M. Peden-Adams, J.M. Keller, D.R. Germolec, Immunotoxicity of perfluorinated compounds: recent developments, Toxicol. Pathol. 40 (2) (2012) 300-311.

[16] G.H. Dong, Y.H. Zhang, L. Zheng, W. Liu, Y.H. Jin, Q.C. He, Chronic effects of perfluorooctanesulfonate exposure on immunotoxicity in adult male C57BL/6 mice, Arch. Toxicol. 83 (9) (2009) 805-815.

[17] K.S. Guruge, H. Hikono, N. Shimada, K. Murakami, J. Hasegawa, L.W. Yeung, N. Yamanaka, N. Yamashita, Effect of perfluorooctane sulfonate (PFOS) on influenza A virus-induced mortality in female B6C3F1 mice, J. Toxicol. Sci. 34 (6) (2009) 687-691.

[18] M.M. Peden-Adams, J.M. Keller, J.G. Eudaly, J. Berger, G.S. Gilkeson, D.E. Keil, Suppression of humoral immunity in mice following exposure to perfluorooctane sulfonate, Toxicol. Sci. 104 (1) (2008) 144-154.

[19] L. Zheng, G.H. Dong, Y.H. Jin, Q.C. He, Immunotoxic changes associated with a 7-day oral exposure to perfluorooctanesulfonate (PFOS) in adult male C57BL/6 mice, Arch. Toxicol. 83 (7) (2009) 679-689.

[20] L. Zheng, G.H. Dong, Y.H. Zhang, Z.F. Liang, Y.H. Jin, Q.C. He, Type 1 and Type 2 cytokines imbalance in adult male C57BL/6 mice following a 7-day oral exposure to perfluorooctanesulfonate (PFOS), J. Immunotoxicol. 8 (1) (2011) $30-38$.

[21] G.H. Dong, J. Wang, Y.H. Zhang, M.M. Liu, D. Wang, L. Zheng, Y.H. Jin, Induction of p53-mediated apoptosis in splenocytes and thymocytes of C57BL/6 mice exposed to perfluorooctane sulfonate (PFOS), Toxicol. Appl. Pharmacol. 264 (2) (2012) 292-299.

[22] Y.H. Zhang, J. Wang, G.H. Dong, M.M. Liu, D. Wang, L. Zheng, Y.H. Jin, Mechanism of perfluorooctanesulfonate (PFOS)-induced apoptosis in the immunocyte, J. Immunotoxicol. 10 (1) (2013) 49-58.

[23] E. Corsini, E. Sangiovanni, A. Avogadro, V. Galbiati, B. Viviani, M. Marinovich, C.L. Galli, M. Dell'Agli, D.R. Germolec, In vitro characterization of the immunotoxic potential of several perfluorinated compounds (PFCs), Toxicol. Appl. Pharmacol. 258 (2) (2012) 248-255.

[24] G.H. Dong, M.M. Liu, D. Wang, L. Zheng, Z.F. Liang, Y.H. Jin, Sub-chronic effect of perfluorooctanesulfonate (PFOS) on the balance of type 1 and type 2 cytokine in adult C57BL6 mice, Arch. Toxicol. 85 (10) (2011) 1235-1244.

[25] J.C. DeWitt, A. Shnyra, M.Z. Badr, S.E. Loveless, D. Hoban, S.R. Frame, R. Cunard, S.E. Anderson, B.J. Meade, M.M. Peden-Adams, R.W. Luebke, M.I. Luster Immunotoxicity of perfluorooctanoic acid and perfluorooctane sulfonate and the role of peroxisome proliferator-activated receptor alpha, Crit. Rev. Toxicol. 39 (1) (2009) 76-94.

[26] M.L. Takacs, B.D. Abbott, Activation of mouse and human peroxisome proliferator-activated receptors (alpha, beta/delta, gamma) by perfluorooctanoic acid and perfluorooctane sulfonate, Toxicol. Sci. 95 (1) (2007) $108-117$.

[27] W.N. Wan Ibrahim, R. Tofighi, N. Onishchenko, P. Rebellato, R. Bose, P. Uhlen, S. Ceccatelli, Perfluorooctane sulfonate induces neuronal and oligodendrocytic differentiation in neural stem cells and alters the expression of PPARgamma in vitro and in vivo, Toxicol. Appl. Pharmacol. 269 (1) (2013) 51-60.

[28] M.R. Qazi, Z. Xia, J. Bogdanska, S.C. Chang, D.J. Ehresman, J.L. Butenhoff, B.D. Nelson, J.W. DePierre, M. Abedi-Valugerdi, The atrophy and changes in the cellular compositions of the thymus and spleen observed in mice subjected to short-term exposure to perfluorooctanesulfonate are high-dose phenomena mediated in part by peroxisome proliferator-activated receptoralpha (PPARalpha), Toxicology 260 (1-3) (2009) 68-76.

[29] M.B. Rosen, J.R. Schmid, J.C. Corton, R.D. Zehr, K.P. Das, B.D. Abbott, C. Lau, Gene expression profiling in wild-type and PPARalpha-null mice exposed to perfluorooctane sulfonate reveals PPARalpha-independent effects, PPAR Res. 2010 (2010). Article ID 794739.

[30] R.E. Mebius, G. Kraal, Structure and function of the spleen, Nat. Rev. Immunol 5 (8) (2005) 606-616.

[31] T. Jia, E.G. Pamer, Immunology: dispensable but not irrelevant, Science (New York, N.Y.) 325 (5940) (2009) 549-550.

[32] L. Cui, Q.F. Zhou, C.Y. Liao, J.J. Fu, G.B. Jiang, Studies on the toxicological effects of PFOA and PFOS on rats using histological observation and chemical analysis, Arch. Environ. Contam. Toxicol. 56 (2) (2009) 338-349.

[33] Y. Wang, L. Wang, Y. Liang, W. Oiu, J. Zhang, O. Zhou, G. Jiang, Modulation of dietary fat on the toxicological effects in thymus and spleen in BALB/c mice exposed to perfluorooctane sulfonate, Toxicol. Lett. 204 (2-3) (2011) $174-182$.

[34] J.C. Liao, R. Boscolo, Y.-L. Yang, L.M. Tran, C. Sabatti, V.P. Roychowdhury, Network component analysis: reconstruction of regulatory signals in biological systems, Proc. Natl. Acad. Sci. 100 (26) (2003) 15522-15527.

[35] M. Kanehisa, S. Goto, S. Kawashima, A. Nakaya, The KEGG databases at GenomeNet,, Nucleic Acids Res. 30 (1) (2002) 42-46.

[36] M.W. Pfaffl, G.W. Horgan, L. Dempfle, Relative expression software tool (REST) for group-wise comparison and statistical analysis of relative expression results in real-time PCR, Nucleic Acids Res. 30 (9) (2002) e36.

[37] M.J. Berridge, M.D. Bootman, P. Lipp, Calcium-a life and death signal, Nature 395 (6703) (1998) 645-648.

[38] J. Fu, Y. Gao, T. Wang, Y. Liang, A. Zhang, Y. Wang, G. Jiang, Elevated levels of perfluoroalkyl acids in family members of occupationally exposed workers: the importance of dust transfer, Sci. Rep. 5 (2015) 9313.

[39] M.R. Qazi, B.D. Nelson, J.W. DePierre, M. Abedi-Valugerdi, 28-Day dietary exposure of mice to a low total dose ( $7 \mathrm{mg} / \mathrm{kg}$ ) of perfluorooctanesulfonate (PFOS) alters neither the cellular compositions of the thymus and spleen nor humoral immune responses: does the route of administration play a pivotal role in PFOS-induced immunotoxicity? Toxicology 267 (2009) 132-139.

[40] P.A. Fair, E. Driscoll, M.A. Mollenhauer, S.G. Bradshaw, S.H. Yun, K. Kannan, G.D. Bossart, D.E. Keil, M.M. Peden-Adams, Effects of environmentallyrelevant levels of perfluorooctane sulfonate on clinical parameters and immunological functions in B6C3F1 mice, J. Immunotoxicol. 8 (1) (2011) $17-29$.

[41] M.A. Mollenhauer, B.J. Carter, M.M. Peden-Adams, G.D. Bossart, P.A. Fair, Gene expression changes in bottlenose dolphin, Tursiops truncatus, skin cells following exposure to methylmercury $(\mathrm{MeHg})$ or perfluorooctane sulfonate (PFOS), Aquat. Toxicol. 91 (1) (2009) 10-18.

[42] M. Malumbres, M. Barbacid, Cell cycle, CDKs and cancer: a changing paradigm, Nat. Rev. Cancer 9 (3) (2009) 153-166.

[43] R.A. Quadri, A. Arbogast, M.A. Phelouzat, S. Boutet, O. Plastre, J.J. Proust, Ageassociated decline in cdk1 activity delays cell cycle progression of human T lymphocytes, J. Immunol. 161 (10) (1998) 5203-5209.

[44] Y. Wang, Y.H. Jin, Perfluorooctane sulfonate (PFOS) and calcium channel downstream signaling molecules, Toxicol. Res. 1 (2) (2012) 103-107.

[45] L. Racioppi, P.K. Noeldner, F. Lin, S. Arvai, A.R. Means, Calcium/calmodulindependent protein kinase kinase 2 regulates macrophage-mediated inflammatory responses, J. Biol. Chem. 287 (14) (2012) 11579-11591.

[46] A. Macho, T. Hirsch, I. Marzo, P. Marchetti, B. Dallaporta, S.A. Susin, N. Zamzami, G. Kroemer, Glutathione depletion is an early and calcium elevation is a late event of thymocyte apoptosis, J. Immunol. 158 (10) (1997) 4612-4619.

[47] A. Strasser, P. Bouillet, The control of apoptosis in lymphocyte selection, Immunol. Rev. 193 (2003) 82-92.

[48] R. Lesourne, S. Uehara, J. Lee, K.D. Song, L. Li, J. Pinkhasov, Y. Zhang, N.P. Weng, K.F. Wildt, L. Wang, R. Bosselut, P.E. Love, Themis, a T cell-specific protein important for late thymocyte development, Nat. Immunol. 10 (8) (2009) $840-847$.

[49] B. Alarcón, S.C. Ley, F. Sanchez-Madrid, R.S. Blumberg, S.T. Ju, M. Fresno, C. Terhorst, The CD3-gamma and CD3-delta subunits of the T cell antigen receptor can be expressed within distinct functional TCR/CD3 complexes, EMBO J. 10 (4) (1991) 903-912.

[50] S. Gelkop, G.D. Gish, Y. Babichev, T. Pawson, N. Isakov, T cell activationinduced CrkII binding to the Zap70 protein tyrosine kinase is mediated by Lck-dependent phosphorylation of Zap70 tyrosine 315, J. Immunol. 175 (12) (2005) 8123-8132.

[51] J.M. Tjon, W.H. Verbeek, Y.M. Kooy-Winkelaar, B.H. Nguyen, A.R. van der Slik, A. Thompson, M.H. Heemskerk, M.W. Schreurs, L.H. Dekking, C.J. Mulder, J. van Bergen, F. Koning, Defective synthesis or association of T-cell receptor chains underlies loss of surface T-cell receptor-CD3 expression in enteropathy-associated T-cell lymphoma, Blood 112 (13) (2008) 5103-5110.

[52] K.A. Anderson, P.K. Noeldner, K. Reece, B.E. Wadzinski, A.R. Means, Regulation and function of the calcium/calmodulin-dependent protein kinase IV/protein serine/threonine phosphatase 2A signaling complex, J. Biol. Chem. 279 (30) (2004) 31708-31716.

[53] H. Enslen, H. Tokumitsu, P.J. Stork, R.J. Davis, T.R. Soderling, Regulation of mitogen-activated protein kinases by a calcium/calmodulin-dependent protein kinase cascade, Proc. Natl. Acad. Sci. U. S. A. 93 (20) (1996) 10803-10808.

[54] B. Liu, H. Barbosa-Sampaio, P.M. Jones, S.J. Persaud, D.S. Muller, The CaMK4/ CREB/IRS-2 cascade stimulates proliferation and inhibits apoptosis of betacells, PLoS One 7 (9) (2012) e45711.

[55] K. Midgett, M.M. Peden-Adams, G.S. Gilkeson, D.L. Kamen, In vitro evaluation of the effects of perfluorooctanesulfonic acid (PFOS) and perfluorooctanoic acid (PFOA) on IL-2 production in human T-cells, J. Appl. Toxicol. 35 (5) (2015) 459-465, http://dx.doi.org/10.1002/jat.3037.

[56] T. Negoro, S. Shimizu, M. Narushima, A.H. Banham, H. Wakabayashi, R. Takayanagi, T. Hagiwara, G. Roncador, T. Osabe, T. Yanai, M. Kin, K. Ikeda, A. Endo, H. Akiyama, Y. Nakano, Elevated receptor for activated C kinase 1 expression is involved in intracellular $\mathrm{Ca}^{2+}$ influx and potentially associated with compromised regulatory $\mathrm{T}$ cell function in patients with asthma, Clin. Exp. Allergy 44 (9) (2014) 1154-1169.

[57] H. Tokumitsu, T.R. Soderling, Requirements for calcium and calmodulin in the calmodulin kinase activation cascade, J. Biol. Chem. 271 (10) (1996) 5617-5622.

[58] G. Huang, L.Z. Shi, H. Chi, Regulation of JNK and p38 MAPK in the immune system: signal integration, propagation and termination, Cytokine 48 (3) (2009) 161-169.

[59] G.L. Johnson, R. Lapadat, Mitogen-activated protein kinase pathways mediated by ERK, JNK, and p38 protein kinases, Science 298 (5600) (2002) 1911-1912.

[60] Y.J. Lee, H.G. Lee, J.H. Yang, Perfluorooctane sulfonate-induced apoptosis of cerebellar granule cells is mediated by ERK $1 / 2$ pathway, Chemosphere 90 (5) (2013) 1597-1602.

[61] X. Shi, B. Zhou, The role of Nrf2 and MAPK pathways in PFOS-induced oxidative stress in zebrafish embryos, Toxicol. Sci. 115 (2) (2010) 391-400.

[62] J.A. McCubrey, M.M. Lahair, R.A. Franklin, Reactive oxygen species-induced activation of the MAP kinase signaling pathways, Antioxid. Redox Signal. 8 (9-10) (2006) 1775-1789. 